\title{
Desempenho de modelos de infiltração na sub-bacia hidrográfica do ribeirão Marcela, na região do alto Rio Grande-MG
}

\section{Performance models of infiltration within the sub-basin of the river Marcela, on the upper Rio Grande-MG}

\author{
Gil Júlio de Souza Netto ${ }^{1 *}$; Antônio Marciano da Silva²; Gilberto Coelho ${ }^{3}$; \\ Dalmo Arantes de Barros ${ }^{4}$; João Carlos Costa Guimarães ${ }^{4}$
}

\section{Resumo}

No Brasil o gerenciamento dos recursos naturais tem sua eficiência comprometida pelo caráter econômico da legislação ambiental vigente. A utilização desses recursos, dentro da agricultura, pecuária e silvicultura, tem desafiado os órgãos gestores de forma a maximizar a produtividade, gerando consequências menos degradantes ao meio ambiente. Diversos são os impactos ambientais significativos gerados pela atividade antrópica, dentre eles, pode-se destacar a erosão do solo. Objetivou-se com este trabalho estimar a infiltração de água no solo por meio de modelos empíricos e teóricos ajustados, por meio de precipitação artificial com diferentes intensidades em parcelas experimentais. A Parcela 1 foi instalada sobre Latossolo Vermelho distrófico típico (LVd) com pastagem; Parcela $2 \mathrm{em}$ Latossolo Vermelho distrófico típico (LVd) ocupada por pastagem nativa; Parcela $3 \mathrm{em}$ Cambissolo distrófico (Cd) ocupada por pastagem nativa degradada com exposição parcial do solo; Parcela 4 em LVAd com pastagem degradada; e Parcela 5 em LVAd ocupada por mata nativa em avançado grau de antropização. Analisando o comportamento da infiltração, observou-se que todos os modelos obtiveram bom ajuste aos dados observados. Todos os solos mostraram semelhança no comportamento da infiltração inicial exceto o Latossolo Vermelho distrófico. Os valores de tempo de saturação ficaram próximos à origem do gráfico para quase todos os eventos de precipitação.

Palavras-chave: Simulador de chuvas, modelagem, intensidade de precipitação

\begin{abstract}
Management of natural resources have been affected by the economical character of present environmental laws applied in Brazil. Use of such resources, especially at agriculture, livestock and forest, has challenged managers in their task to maximize yield less devastating to environment. Various are the impacts brought by human activities and an important one is soil erosion. At this research, water infiltration in soils were evaluated by empirical methods and theoretical approaches, by simulating precipitation with different intensities at experimental plots. Plot 1 was installed at a dark red latosol (LVd) with pasture; plot 2 at same soil but covered with native pasture; plot 3 at distrofic cambisoil
\end{abstract}

${ }^{1}$ Eng $^{\circ}$ Agrícola, M.e em Engenharia Agrícola, Dept ${ }^{\circ}$ de Engenharia, DEG, Universidade Federal de Lavras, UFLA, Lavras, MG. E-mail: gil_netto1984@hotmail.com

${ }^{2}$ Eng $^{\mathrm{o}}$ Florestal, Dr. Engenharia Hídráulica, Prof. Titular do Dept ${ }^{\circ}$ de Engenharia/DEG, UFLA, Lavras, MG. E-mail: marciano@ deg.ufla.br

${ }^{3}$ Eng $^{\circ}$ Agrícola, Dr. Recursos Hídricos, Prof. Adjunto, Dept ${ }^{\circ}$ de Engenharia/DEG, UFLA, Lavras, MG. E-mail: coelho@deg.ufla. br

${ }^{4}$ Eng $^{\mathrm{os}_{\mathrm{s}}}$ Florestal, Discentes de Doutorado em Engenharia Florestal, Dept ${ }^{\mathrm{o}}$ de Ciências Florestais/DCF, UFLA, Lavras, MG. E-mail: dalmo.barros@uol.com.br; joao.guimaraes77@gmail.com

* Autor para correspondência 
(Cd) with native pasture but significantly devastated. Plot 4 was in a red yellow latosol with devastated pasture and plot 5, at same soil, covered with devastated forest. Through infiltration behavior analysis, it could be observed that all models fitted well the observed values of infiltration. When initial infiltration was analyzed separately, distrofic red latosol was different. Soil saturation time was close to graphic origin for all precipitation events.

Key words: Rain simulator, modeling, rain intensity

\section{Introdução}

No Brasil o gerenciamento dos recursos naturais tem sua eficiência comprometida pelo caráter econômico da legislação ambiental vigente. A utilização desses recursos, dentro da agricultura, pecuária e silvicultura, tem desafiado os órgãos gestores de forma a maximizar a produtividade, gerando consequências menos degradantes ao meio ambiente. Neste sentido, diversos são os impactos ambientais significativos gerados pela atividade antrópica, dentre eles, pode-se destacar a erosão do solo.

O processo de erosão hídrica do solo envolve a desagregação de partículas do solo pelo impacto das gotas de chuva, o transporte e consequente deposição pelo escoamento superficial. Este escoamento ocorre na forma de fluxo laminar raso o que caracteriza a erosão (BEZERRA; CANTALICE, 2009; SILVA et al., 2010). Este processo torna-se mais intenso quando influenciado pela declividade e agrava-se pelas ações antrópicas (SILVA et al., 2010).

A erosão do solo é um fenômeno cujo impacto sobre os recursos naturais renováveis tem preocupado governos e instituições de todo o mundo. $\mathrm{O}$ enfoque principal deste fenômeno tem sido considerado uma ameaça à própria existência da humanidade. Essa ameaça tem contribuído para uma imediata necessidade de se quantificar e controlar, de forma racional, as causas dos processos erosivos. A demanda por novas metodologias capazes de avaliar com precisão a estimativa das perdas de solo e água tem resultado um grande número de pesquisas científicas (SILVA et al., 2010).

Uma vez que a erosão é um processo predominantemente de superfície, as condições físicas da camada superficial do solo, em última análise são as que irão determinar as perdas finais de solo e água. Basicamente, essas condições dependem do tipo de uso da terra e da forma como o solo é manejado, induzindo diferentes graus de cobertura, rugosidade e consolidações superficiais, bem como diferentes características físicas e químicas do solo (BAGATINI et al., 2011).

Segundo Panachuki et al. (2011), as operações de preparo do solo influenciam fortemente a erosão hídrica das áreas cultivadas, alterando o micro relevo e a cobertura por resíduos vegetais, promovendo a exposição da superfície do solo à ação da chuva e consequentemente, do escoamento superficial. Estes autores consideram também que o micro relevo do solo, caracterizado pela rugosidade de sua superfície, induzido pelas operações de preparo é de fundamental importância na retenção e infiltração de água no solo, elevando a capacidade de armazenamento depressional de água na superfície e, com isso, diminuindo o escoamento superficial.

A infiltração é definida como a passagem de água da superfície para o interior do solo, configurando-se entre os mais importantes processos que compõem o ciclo hidrológico, sendo um fator determinante da disponibilização de água para as culturas, da recarga dos aquíferos subterrâneos, da ocorrência e magnitude do escoamento superficial e do manejo do solo e da água (CECÍLIO et al., 2003).

De acordo com Urchei e Fietz (2002), a taxa de infiltração é o volume de água que atravessa a unidade de área da superfície do solo por unidade de tempo, a qual tende a decrescer, atingindo um valor final constante, denominado taxa de infiltração básica (TIB). A magnitude da TIB é de grande 
importância para a escolha e dimensionamento do manejo de sistemas de irrigação e drenagem. Em virtude da natureza dos processos, das características ambientais, do método utilizado para determinação e o tipo e manejo do solo, a taxa de infiltração apresenta grande variabilidade.

Estudos sobre o efeito das chuvas em atributos do solo são complexos de serem realizados com chuva natural, pois não se tem controle sobre a duração, intensidade, distribuição e tipo de chuva. Uma alternativa que se apresenta é a utilização de simuladores de chuvas que permitem controlar as suas características. Os simuladores têm a vantagem de poderem ser utilizados a qualquer momento e em qualquer situação. Este tipo de equipamento já vem sendo utilizado em estudos de manejo de solos há bastante tempo. Já foram construídos vários modelos e atualmente existem alguns totalmente informatizados (FERREIRA et al., 2010).

Neste contexto, Pinheiro, Teixeira e Kaufmann (2009) afirmam que a compreensão e a avaliação da capacidade de armazenamento de água no solo, bem como os fluxos que ocorrem tanto na superfície (infiltração e evaporação) quanto em profundidade no solo (drenagem interna), são importantes quando se trata da irrigação, drenagem, erosão, biologia da fauna e flora, lixiviação de elementos químicos, poluição do solo e da água, entre outros aspectos. Porém, caracterizar todas as variáveis que influenciam a infiltração é um processo complexo.

A intensidade com que algumas variáveis afetam o processo de infiltração ainda hoje, não é bem definida. Sendo assim, diversos modelos têm sido propostos visando simplificar o estudo da infiltração da água no solo. Os solos apresentam grande variabilidade espacial, ainda que dentro de pequenas áreas. Como resultado disto e também das variações temporais das propriedades do solo, a infiltração é um processo muito dinâmico, que pode ser descrito apenas de forma aproximada, mediante o uso de equações matemáticas (CECÍLIO et al., 2003).
O processo de infiltração de água no solo pode ser descrito por diversas equações ou modelos, alguns desenvolvidos a partir de considerações físicas, enquanto outros de forma empírica. Um modelo empírico muito empregado em manejo de irrigação é a equação de Kostiakov-Lewis, normalmente utilizado para a estimativa da infiltração acumulada, cujos parâmetros não tem significado físico próprio e são estimados a partir de dados experimentais. O modelo de Horton (HORTON, 1940), o qual também é um modelo empírico, é considerado mais consistente que o de Kostiakov-Lewis, é escrito na forma de uma função exponencial (DALRI et al., 2010).A maioria dos modelos que se propõem a descrever o processo de infiltração, incluindo-se o de Green-Ampt (GREEN; AMPT, 1911), parte do pressuposto que o perfil de solo no qual se processa a infiltração é homogêneo, o que, na grande maioria dos casos, não se constitui em uma verdade; portanto, o estudo do processo de infiltração em solos estratificados torna-se essencial para uma predição mais correta, tanto da quantidade de água infiltrada no solo, como da taxa com que se dá o processo (CECÍLIO et al., 2003).

Sendo assim, objetivou-se com este trabalho avaliar o desempenho de modelos preditivos de infiltração da água no solo, relacionando os resultados encontrados com as características de uso e ocupação do solo em uma sub-bacia hidrográfica do Alto Rio Grande, situada a montante da Usina Hidroelétrica (UHE) de Camargos/Cemig, MG.

\section{Material e Métodos}

Para elaboração do ensaio proposto, os estudos foram concentrados em uma área pertencente àsubbacia hidrográfica do ribeirão Marcela, situada entre as coordenadas UTM 550169 e 552810 O e 7650163 e 7650989 S. De acordo com Giarola et al. (1997), a região está inserida na unidade geomorfológica Planalto Campo das Vertentes, Alto Rio Grande, Sul do estado de Minas Gerais. O clima da região é do tipo Cwa, temperado com verões quentes e úmidos 
e invernos secos e frios, utilizando a classificação de Köppen (1936).

Na sub-bacia em questão ocorre a predominância do Latossolo Vermelho Amarelo distrófico (LVAd), ocupando cerca de $65 \%$ da área, enquanto o Latossolo Vermelho distrófico típico (LVd) ocupa cerca de 14\%. Os Cambissolos (Cd) estão presentes em menos de 5\% e os solos Hidromórficos (Hi) em $17 \%$ da área da sub-bacia. Com base em estudos pedológicos e hidrológicos, a área foi considerada por Beutler et al. (2001) e Silva et al. (2006) como representativa do domínio dos Latossolos na região Alto Rio Grande à montante da UHE de Camargos/ CEMIG.

Na sub-bacia analisada, o uso do solo concentrase na pecuária extensiva, justificando a presença de pastagem em aproximadamente $75 \%$ da área. Entretanto, observa-se, de forma menos expressiva, a presença de lavouras de milho, arroz, cana-deaçúcar e fruticultura tropical, além de pequenos e antropizados fragmentos de florestas nativas, bem como extensas áreas de eucalipto. Este cenário pouco modificou durante o período do estudo.

O perímetro da bacia hidrográfica é de $8,94 \mathrm{~km}$, o coeficiente de compacidade $(\mathrm{kc})$ igual a 1,14 , sendo considerada uma bacia com alta propensão a elevadas vazões de pico. De acordo com o coeficiente de forma $(\mathrm{kf}=0,79)$ a bacia tem tendência mediana à formação de enchentes. A sub-bacia possui uma área de drenagem de aproximadamente 478 ha, com declividade média de $13,65 \%$, e altitude média de $1.003 \mathrm{~m}$. O comprimento do curso d'água principal é de 3,83 km sendo a declividade do canal de 1,57\% e a densidade de drenagem igual a $1,80 \mathrm{~km} . \mathrm{km}^{-2}$ (GIAROLA et al., 1997).

O monitoramento das parcelas ocorreu durante o período de setembro de 2009 a abril de 2010 . As parcelas experimentais para avaliação da infiltração e perda de solo na sub-bacia hidrográfica apresentaram área de 1,0 $\mathrm{m}^{2}$ e foram distribuídas aleatoriamente nas diversas unidades pedológicas da sub-bacia, visando garantir a representatividade para cada evento pluviométrico simulado.

Foram instaladas cinco parcelas na área de acordo com as características elencadas por Silva (2006): parcela 1 (P1) - Latossolo Vermelho Distrófico típico $(\mathrm{LVd})$ em área com declividade de $10 \%$, sob pastagem degradada; parcela 2 (P2) Latossolo Vermelho Distrófico típico (LVd) em área com declividade de $13 \%$ sob vegetação de pastagem nativa conservada; parcela 3 (P3) - Cambissolo distrófico $(\mathrm{Cd})$ em área com declividade de 15\% sob pastagem degradada; parcela 4 (P4) - Latossolo Vermelho Amarelo distrófico (LVAd) em área com declividade de $10 \%$ e coberta por pastagem, com pouca exposição do solo; e parcela 5 (P5) - Latossolo Vermelho Amarelo distrófico (LVAd) em área com declividade de $15 \%$ sob mata nativa antropizada.

O equipamento simulador de chuva utilizado foi composto por um gerador de energia elétrica Branco B4T 5000 L, uma Motobomba Schneider 1,0 cv; e um Simulador de Chuvas portátil, desenvolvido no Laboratório de Hidráulica da Universidade Federal de Lavras.

Durante as simulações, para todos os testes, manteve-se arbitrariamente uma hora de precipitação simulada para cada evento, contados a partir do início do escoamento superficial. As leituras foram tomadas a cada cinco minutos e serviram como base para processamento e ajuste dos parâmetros das equações de infiltração.

As intensidades de precipitação utilizadas foram de $30 \mathrm{~mm} \mathrm{~h}^{-1}, 60 \mathrm{~mm} \mathrm{~h}^{-1}, 90 \mathrm{~mm} \mathrm{~h}^{-1}, 120 \mathrm{~mm} \mathrm{~h}^{-1}, 150$ $\mathrm{mm} \mathrm{h}^{-1}$ vinculadas aos microaspersores utilizados no simulador de chuva, cuja uniformidade de distribuição fornecida pelo fabricante foi avaliada em testes feitos em laboratório com valores dos coeficientes de uniformidade de Christiansen (CUC) maiores que $92 \%$.

Toda área lateral e superior do simulador de chuvas foi coberta por uma capa plástica durante a simulação, a fim de evitar possíveis interferências da ação dos ventos e da temperatura sobre as pequenas partículas de água aspergidas pelos microaspersores. 
Os modelos empíricos utilizados neste estudo foram os de Kostiakov, Kostiakov-Lewis, Horton e o modelo teórico de Green-Ampt. Entre as equações empíricas mais utilizadas para fins de dimensionamento de sistemas hidroagrícolas, a desenvolvida por Kostiakov (1932) possui grande abrangência (1):

$$
I=K * t^{\alpha}
$$

Onde:

$I=$ infiltração acumulada no tempo t (L);

$t=$ tempo decorrido desde o início do processo $(\mathrm{T})$;

$K=$ capacidade de infiltração no tempo $\mathrm{t}=1$;

$\alpha=$ parâmetro característico do solo, representa a taxa de decréscimo de I em função do tempo.

A equação de Kostiakov é imprópria tanto para $t$ $=0$ quanto para $\mathrm{t} \rightarrow \infty$, pois, os valores resultantes nestas condições são irreais, caracterizando descontinuidade. No entanto, o modelo de Kostiakov-Lewis foi desenvolvido para suprir essa herança matemática deixada pelo modelo de Kostiakov. Para um tempo tendendo ao infinito, a equação não mais tende à zero, e, sim, para infiltração básica de acordo com a equação (2):

$$
I=K * t^{\alpha}+I_{b} * t
$$

Onde:

$I=$ infiltração acumulada no tempo t (L);

$K=$ capacidade de infiltração no tempo $\mathrm{t}=1$;

$t=$ tempo decorrido desde o início do processo (T);

$\alpha=$ parâmetro característico do solo - taxa de decréscimo de I em função do tempo;

$I_{b}=$ capacidade de infiltração básica do solo $\left(\right.$ L. $\left.T^{-1}\right)$.

Uma opção bastante aceita por sua simplicidade é a Equação de Horton (3) (HORTON, 1940). É uma equação empírica, na qual se assume que a infiltração inicia com uma taxa $I_{i}$ e decresce exponencialmente com o tempo t. Depois de um tempo variável, quando a umidade do solo atinge um grau elevado (próximo da saturação), a taxa de infiltração converge para um valor constante $I_{b}$.

$$
I=I_{b} \cdot t+\frac{\left(I_{i}-I_{b}\right)}{K} \cdot\left(1-e^{-K}\right)
$$

Onde:

$I=$ infiltração acumulada no tempo t (L);

$I_{b}=$ valor inicial de CI, para $\mathrm{t}=0\left(\mathrm{~L}^{\mathrm{T}} \mathrm{T}^{-1}\right)$;

$t=$ tempo decorrido desde o início do processo (T);

$I_{i}=$ valor para o qual tende CI, quando t muito grande $\left(\mathrm{L} . \mathrm{T}^{-1}\right)$;

$K=$ constante característica do solo.

O modelo de infiltração de Green-Ampt (GREEN; AMPT, 1911), muito utilizado ainda hoje em Hidrologia, não permite explicitar de forma exata o volume acumulado por infiltração em função do tempo.

$I(t)=K_{s} \cdot t+\psi_{f} \cdot\left(\theta_{s}-\theta_{i}\right) \cdot \mathrm{h} \cdot\left[1+\frac{I(t)}{\psi_{f} \cdot\left(\theta_{s}-\theta_{i}\right)}\right]$

Onde:

$I(t)=$ infiltração acumulada $(\mathrm{L})$;

$t_{i}=$ taxa de infiltração da água no solo (L.T $\left.{ }^{-1}\right)$;

$K_{s}=$ condutividade hidráulica do solo saturado (L. $\left.\mathrm{T}^{-1}\right)$;

$\psi_{f}=$ potencial matricial médio na frente de umedecimento (L);

$\theta_{s}=$ umidade do solo na saturação $\left(\mathrm{L}^{3} \cdot \mathrm{L}^{-3}\right)$;

$\theta_{i}=$ umidade inicial do solo $\left(\mathrm{L}^{3} \cdot \mathrm{L}^{-3}\right)$.

A infiltração acumulada (I) é dada pela equação: 


$$
I=Z \cdot\left(\theta_{s}-\theta_{i}\right)
$$

Onde:

$I=$ infiltração acumulada no tempo t (L);

$Z=$ profundidade da frente de umedecimento $(\mathrm{mm})$;

$\theta_{s}=$ umidade do solo na saturação $\left(\mathrm{L}^{3} \cdot \mathrm{L}^{-3}\right)$;

$\theta_{i}=$ umidade inicial do solo $\left(\mathrm{L}^{3} \cdot \mathrm{L}^{-3}\right)$.

Para todas as parcelas e intensidades de precipitação, foram ajustados os parâmetros dos modelos empíricos e teóricos de infiltração utilizados de acordo com o método de GaussNewton. A análise estatística dos dados quantitativos obtidos neste estudo foi feita por meio de análise de variância.

Em cada evento simulado de chuva, o monitoramento da umidade foi feito em dois momentos distintos, antes e imediatamente após o evento, sendo a umidade determinada pelo método gravimétrico.

As amostras deformadas de solo foram coletadas em cada parcela, por meio de trado manual. O material coletado foi colocado em latas identificadas, nas profundidades de 0 a $0,20 \mathrm{~m}$ e 0,20 a $0,40 \mathrm{~m}$ para a determinação da umidade. As umidades, inicial e final, em base volumétrica, foram obtidas multiplicando a umidade em peso base seca pela densidade do solo de cada parcela conforme os dados de atributos físico-hídricos extraídos de Gomes (2005).

\section{Resultados e Discussão}

De modo geral, os coeficientes de determinação $\left(\mathrm{R}^{2}\right)$, para todos os modelos de infiltração ajustados foram superiores a 0,92 , indicando que todos os modelos são satisfatórios na previsão da infiltração básica de água no solo, corroborando com Paixão et al. (2009), que obtiveram para todos os modelos e tipos de solo avaliados, resultados maiores que 0,95 . Os parâmetros ajustados para o modelo de Kostiakov e seus valores variaram conforme Tabela 1.

Analisando o parâmetro $\alpha, \quad$ o intervalo ficou próximo do linear para todos os eventos de precipitação simulados sobre diferentes condições de solos, mostrando neste comportamento influência da umidade inicial e do uso e ocupação do solo na sub-bacia, neste caso, $75,87 \%$ de pasto. 
Tabela1. Relação entre tipo de solo, cobertura vegetal, declive, intensidade de precipitação (IP), parâmetros (K e $\alpha$ ) da equação de Kostiakov e umidade inicial ( $\theta i)$ com o índice de representatividade do ajuste $\left(\mathrm{R}^{2}\right)$.

\begin{tabular}{|c|c|c|c|c|}
\hline \multicolumn{5}{|c|}{ LVd com pastagem natural e $10 \%$ de declive (P1) } \\
\hline IP & $\mathbf{K}$ & $\alpha$ & $\mathbf{R}^{2}$ & $\theta \mathrm{i}\left(\mathrm{cm}^{3} \cdot \mathrm{cm}^{-3}\right)$ \\
\hline 30 & 0,5003 & 0,9829 & 1,0000 & 0,3532 \\
\hline 60 & 0,8989 & 0,9786 & 0,9999 & 0,4232 \\
\hline 90 & 1,3900 & 0,9900 & 1,0000 & 0,1802 \\
\hline 120 & 1,9200 & 0,9900 & 1,0000 & 0,1802 \\
\hline 150 & 2,3129 & 0,9800 & 0,9998 & 0,3185 \\
\hline \multicolumn{5}{|c|}{ LVd com pastagem natural e $13 \%$ de declive (P2) } \\
\hline 30 & 0,6713 & 0,8965 & 0,9988 & 0,3815 \\
\hline 60 & 1,1971 & 0,9359 & 1,0000 & 0,4708 \\
\hline 90 & 2,6437 & 0,7886 & 0,9438 & 0,3725 \\
\hline 120 & 2,5532 & 0,9196 & 0,9999 & 0,3376 \\
\hline 150 & 3,9137 & 0,8424 & 0,9996 & 0,4142 \\
\hline \multicolumn{5}{|c|}{ Cd com pastagem natural degradada e $15 \%$ de declive (P3) } \\
\hline 30 & 0,5829 & 0,9426 & 0,9998 & 0,3995 \\
\hline 60 & 1,1171 & 0,9342 & 1,0000 & 0,3767 \\
\hline 90 & 1,7092 & 0,9374 & 1,0000 & 0,4505 \\
\hline 120 & 2,3847 & 0,9404 & 1,0000 & 0,3290 \\
\hline 150 & 2,5962 & 0,9800 & 0,9991 & 0,2601 \\
\hline \multicolumn{5}{|c|}{ LVAd com pastagem natural e $10 \%$ de declive (P4) } \\
\hline 30 & 0,7273 & 0,8900 & 0,9993 & 0,4390 \\
\hline 60 & 1,3261 & 0,9200 & 0,9997 & 0,3748 \\
\hline 90 & 1,5507 & 0,9879 & 1,0000 & 0,3594 \\
\hline 120 & 2,1273 & 0,9819 & 1,0000 & 0,3281 \\
\hline 150 & 2,5373 & 0,9903 & 1,0000 & 0,2333 \\
\hline \multicolumn{5}{|c|}{ LVAd com pastagem natural e $15 \%$ de declive (P5) } \\
\hline 30 & 0,5800 & 0,9400 & 0,9998 & 0,5351 \\
\hline 60 & 1,3770 & 0,9100 & 0,9987 & 0,4700 \\
\hline 90 & 2,7230 & 0,8400 & 0,9902 & 0,4266 \\
\hline 120 & 2,4871 & 0,9500 & 0,9999 & 0,4218 \\
\hline 150 & 2,6933 & 0,9800 & 1,0000 & 0,3807 \\
\hline
\end{tabular}

Fonte: Elaboração dos autores.

Observou-se também uma correlação entre a umidade inicial e os valores do parâmetro $\alpha$, onde os menores valores de umidade se correlacionaram aos maiores valores de $\alpha$, excetuando para o LVd. Comparativamente aos outros modelos observou-se que o modelo de Kostiakov não foi o que melhor se ajustou às condições dos solos avaliados divergindo com as observações de Cunha et al. (2009). Pode-se atribuir estas mudanças de comportamento às características de simulação de chuvas como tamanho da parcela, deriva gotas gerando desuniformidade de aplicação, da altura dos microaspersores e dos diferentes declives e coberturas vegetais. Os parâmetros ajustados para o modelo de Kostiakov-Lewis e seus valores variaram conforme Tabela 2. 
Tabela 2. Relação entre tipo de solo, cobertura vegetal, declive, intensidade de precipitação (IP) e os parâmetros capacidade de infiltração (K), parâmetro característico do solo $(\alpha)$ e infiltrabilidade básica(Ib) da equação de KostiakovLewis das parcelas 1 a 5 com o índice de representatividade do ajuste $\left(\mathrm{R}^{2}\right)$.

\begin{tabular}{|c|c|c|c|c|c|c|}
\hline Parcela & IP $\left(\mathrm{mm} \mathrm{h}^{-1}\right)$ & $\mathbf{K}\left(\mathrm{mm} \mathrm{h}^{-1}\right)$ & $\alpha$ & Ib $\left(\mathrm{mm} \mathrm{h}^{-1}\right)$ & $\mathbf{R}^{2}$ & $\theta \mathrm{i}\left(\mathrm{cm}^{3} \cdot \mathrm{cm}^{-3}\right)$ \\
\hline \multirow{5}{*}{$\begin{array}{c}\text { LVd, } \mathrm{PN} \mathrm{e} \\
10 \% \text { de declive } \\
(\mathrm{P} 1)\end{array}$} & 30 & 0,1000 & 0,5000 & 0,4529 & 0,9957 & 0,3532 \\
\hline & 60 & 0,2350 & 0,8840 & 0,6775 & 0,9999 & 0,4232 \\
\hline & 90 & 0,4280 & 0,9629 & 0,9696 & 0,9998 & 0,1802 \\
\hline & 120 & 0,9289 & 0,9610 & 0,9921 & 0,9997 & 0,1802 \\
\hline & 150 & 0,9426 & 0,9500 & 0,8623 & 0,9986 & 0,3185 \\
\hline \multirow{5}{*}{$\begin{array}{c}\text { LVd, PN e } \\
13 \% \text { de declive } \\
(\mathrm{P} 2)\end{array}$} & 30 & 0,4100 & 0,6800 & 0,3319 & 0,9993 & 0,3815 \\
\hline & 60 & 0,8952 & 0,8977 & 0,3014 & 1,0000 & 0,4708 \\
\hline & 90 & 3,4874 & 0,3810 & 0,8538 & 0,9441 & 0,3725 \\
\hline & 120 & 1,7252 & 0,8385 & 0,9457 & 0,9999 & 0,3376 \\
\hline & 150 & 3,3254 & 0,7271 & 0,9700 & 0,9995 & 0,4142 \\
\hline \multirow{5}{*}{$\begin{array}{c}\text { Cd, PND e } \\
15 \% \text { de declive } \\
\text { (P3) }\end{array}$} & 30 & 0,1000 & 0,5000 & 0,4529 & 1,0000 & 0,3995 \\
\hline & 60 & 0,7014 & 0,8698 & 0,4422 & 1,0000 & 0,3767 \\
\hline & 90 & 1,2520 & 0,9056 & 0,4727 & 1,0000 & 0,4505 \\
\hline & 120 & 1,8433 & 0,9149 & 0,5672 & 1,0000 & 0,3290 \\
\hline & 150 & 2,6401 & 0,8961 & 0,6948 & 0,9989 & 0,2601 \\
\hline \multirow{5}{*}{$\begin{array}{c}\text { LVAd, PN e } \\
13 \% \text { de declive } \\
(\mathrm{P} 4)\end{array}$} & 30 & 0,0297 & 0,5760 & 0,4760 & 1,0000 & 0,4390 \\
\hline & 60 & 0,0199 & 0,7500 & 0,9719 & 0,9988 & 0,3748 \\
\hline & 90 & 0,2838 & 0,9121 & 1,2778 & 0,9926 & 0,3594 \\
\hline & 120 & 0,7248 & 0,9383 & 1,4125 & 1,0000 & 0,3281 \\
\hline & 150 & 0,2144 & 0,7256 & 2,3700 & 1,0000 & 0,2333 \\
\hline \multirow{5}{*}{$\begin{array}{l}\text { LVAd, MN e } \\
15 \% \text { de declive } \\
\text { (P5) }\end{array}$} & 30 & 0,1000 & 0,5000 & 0,4529 & 1,0000 & 0,5351 \\
\hline & 60 & 1,2000 & 0,8864 & 0,2085 & 0,9988 & 0,4700 \\
\hline & 90 & 0,5500 & 0,0389 & 1,4013 & 0,9926 & 0,4266 \\
\hline & 120 & 1,0000 & 0,3000 & 1,9491 & 1,0000 & 0,4218 \\
\hline & 150 & 0,8000 & 0,4300 & 2,4035 & 1,0000 & 0,3807 \\
\hline
\end{tabular}

Fonte: Elaboração dos autores.

Por meio do ajuste dos dados pelo modelo de Kostiav-Lewis, observou-se no Latossolo Vermelho Amarelo distrófico valores muito baixos no parâmetro $\alpha$ sob intensidade de precipitação de 90 $\mathrm{mm} \mathrm{h}^{-1}$. Este fato pode estar associado à cobertura vegetal de mata nativa.

Os valores de umidade inicial em ambas foram muito semelhantes podendo associar o intervalo de $42 \%$ a $43 \%$ como favorável à variação do parâmetro $\alpha$ para estes tipos de solos confirmando as observações de Bernardo, Soares e Mantovani (2006), que a umidade inicial é o principal fator responsável pela variação da taxa de infiltração inicial. Equivalente ao modelo de Kostiakov, o modelo de Kostiakov-Lewis ajustou os parâmetros com tendências lineares assim como o modelo de Horton conforme Tabela 3. 
Tabela 3. Relação entre tipo de solo, cobertura vegetal, declive, intensidade de precipitação (IP) e os parâmetros capacidade de infiltração (K), infiltrabilidade básica (Ib), e Infiltração inicial (Ii) da equação de Horton com o índice de representatividade do ajuste $\left(\mathrm{R}^{2}\right)$.

\begin{tabular}{|c|c|c|c|c|c|}
\hline Parcela & IP $\left(\mathrm{mm} \mathrm{h}^{-1}\right)$ & $\mathbf{K}\left(\mathrm{mm} \mathrm{h}^{-1}\right)$ & Ib $\left(\mathrm{mm} \mathrm{h}^{-1}\right)$ & Ii $\left(\mathrm{mm} \mathrm{h}^{-1}\right)$ & $\mathbf{R}^{2}$ \\
\hline \multirow{5}{*}{$\begin{array}{l}\text { LVd, PN e } \\
10 \% \text { de } \\
\text { declive } \\
\text { (P1) }\end{array}$} & 30 & 1,0131 & 0,4583 & 0,8865 & 0,9957 \\
\hline & 60 & 0,5308 & 0,8152 & 1,1142 & 0,9999 \\
\hline & 90 & 0,5857 & 1,3309 & 1,5875 & 0,9998 \\
\hline & 120 & 0,9575 & 1,8453 & 2,2679 & 0,9997 \\
\hline & 150 & 0,8884 & 2,0910 & 3,5984 & 0,9986 \\
\hline \multirow{5}{*}{$\begin{array}{l}\text { LVd, PN e } \\
13 \% \text { de } \\
\text { declive } \\
\text { (P2) }\end{array}$} & 30 & 0,5487 & 0,4532 & 0,5123 & 0,9977 \\
\hline & 60 & 0,9697 & 0,8893 & 2,7946 & 0,9999 \\
\hline & 90 & 0,8747 & 1,0226 & 7,4883 & 0,9219 \\
\hline & 120 & 0,8977 & 1,7422 & 6,7476 & 0,9998 \\
\hline & 150 & 1,0203 & 1,8609 & 13,5506 & 0,9999 \\
\hline \multirow{5}{*}{$\begin{array}{l}\text { Cd, PND e } \\
15 \% \text { de } \\
\text { declive } \\
\text { (P3) }\end{array}$} & 30 & 3,1872 & 0,4724 & 0,6854 & 1,0000 \\
\hline & 60 & 0,0761 & 0,8057 & 1,0284 & 1,0000 \\
\hline & 90 & 0,0510 & 1,2255 & 1,5354 & 1,0000 \\
\hline & 120 & 0,9417 & 1,7905 & 5,9656 & 1,0000 \\
\hline & 150 & 2,3065 & 2,3912 & 3,5677 & 0,9992 \\
\hline \multirow{5}{*}{$\begin{array}{l}\text { LVAd, PN e } \\
13 \% \text { de } \\
\text { declive } \\
\text { (P4) }\end{array}$} & 30 & 0,7252 & 0,4803 & 0,5328 & 1,0000 \\
\hline & 60 & 0,9994 & 0,9784 & 1,0294 & 1,0000 \\
\hline & 90 & 1,0437 & 1,4628 & 2,2305 & 1,0000 \\
\hline & 120 & 1,1980 & 1,9536 & 3,4922 & 1,0000 \\
\hline & 150 & 2,2808 & 2,4315 & 3,7739 & 1,0000 \\
\hline \multirow{5}{*}{$\begin{array}{l}\text { LVAd, MN } \\
\text { e } 15 \% \text { de } \\
\text { declive } \\
\text { (P5) }\end{array}$} & 30 & 0,6697 & 0,4659 & 0,5302 & 1,0000 \\
\hline & 60 & 0,4665 & 0,9608 & 1,0138 & 0,9994 \\
\hline & 90 & 0,9120 & 1,2234 & 7,8005 & 0,9926 \\
\hline & 120 & 1,3863 & 1,9743 & 3,6345 & 1,0000 \\
\hline & 150 & 2,7292 & 2,4691 & 3,1607 & 1,0000 \\
\hline
\end{tabular}

Fonte: Elaboração dos autores.

Pelo modelo de Horton observou-se que a diferença entre a taxa de infiltração inicial e a infiltração básica propiciou um decréscimo muito rápido na taxa de infiltração, que pode estar ligado, entre outros fatores, ao manejo do solo. Esta observação corrobora com Weiler e Naef (2003) que também encontraram um decréscimo muito rápido na taxa de infiltração em função da elevada compactação proporcionada pelo pisoteio animal durante o pastejo. Procurou-se avaliar também a correlação entre a intensidade de precipitação e a infiltração inicial conforme Figura 1. Nela procurouse avaliar por meio de linha de tendência correlação entre os coeficientes das equações geradas e os diferentes tipos de solos da sub-bacia.

Para todos os solos da sub-bacia, os coeficientes angulares indicaram boa correlação excetuando o Latossolo Vermelho distrófico típico com cobertura vegetal de pastagem nativa e $13 \%$ de declividade. A variação existente entre os coeficientes podem estar ligados às diferentes condições de umidade inicial, uso e cobertura e declive. Isto reforça ainda mais o peso que a variabilidade espacial dos atributos físicos e hídricos do solo exerce sobre a o processo de infiltração dentro de uma sub-bacia. 
Figura 1. Linearização da relação entre a Intensidade de Precipitação (IP) - eixo (x) - e Infiltração Inicial (Ii) - eixo (y) - pelo modelo de Horton $(\mathrm{H})$ para todos os solos, uso e ocupação e declive (i) com o índice de representatividade do ajuste $\left(\mathrm{R}^{2}\right)$.

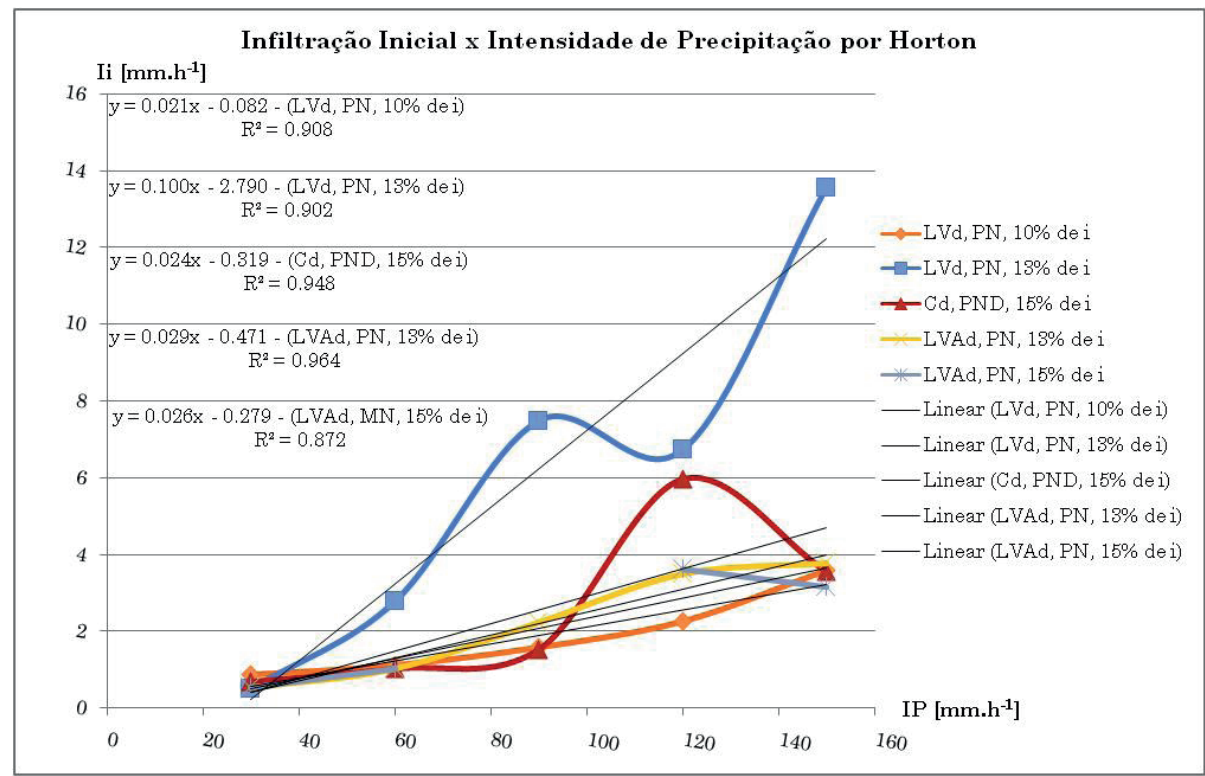

Fonte: Elaboração dos autores.

Vale ressaltar que quando a pesquisa incorpora os atributos físicos e hídricos do solo, espera-se obter correlações de dados de infiltração em função da intensidade de precipitação. Por meio da Figura
2, procurou-se correlacionar o tempo de saturação (Time Pound) dos modelos de Kostiakov-Lewis e Horton de modo avaliar a variabilidade dos valores ajustados pelos modelos.

Figura 2. Relação entre os tempos de saturação pelos modelos de Kostiakov-Lewis e Horton com o índice de representatividade do ajuste $\left(\mathrm{R}^{2}\right)$.

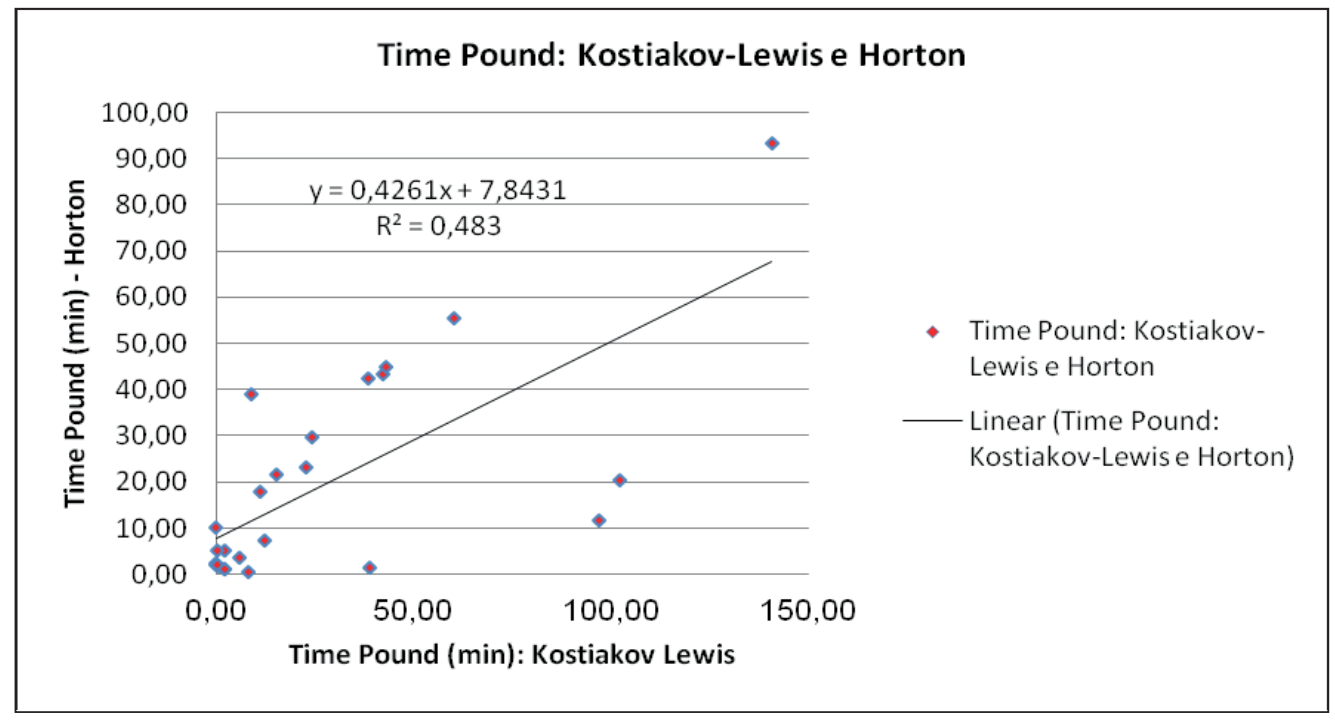

Fonte: Elaboração dos autores. 
Observou-se que os valores ajustados, para ambos os modelos, atingiram o tempo de saturação próximo a origem podendo estar ligados aos valores de umidade inicial corroborando com as observações de Bernardo, Soares e Mantovani (2006). O coeficiente de determinação da equação, considerando a alta variabilidade espacial e temporal dos atributos físicos e hídricos do solo, associados às particularidades de cada modelo, forneceu boa correlação entre os tempos de saturação de Kostiakov-Lewis e para Horton.

Os maiores tempos calculados pelo modelo de Kostiakov-Lewis foram para o Cambissolo distrófico, com pastagem natural degradada, e, para Latossolos Vermelho Amarelo distrófico, com mata nativa, atingindo valores de $01 \mathrm{~min} 37,07 \mathrm{~s}$ e $02 \mathrm{~min}$ $20,79 \mathrm{~s}$, para as intensidades de $150 \mathrm{~mm} \mathrm{~h}^{-1}$ e 120 $\mathrm{mm} \mathrm{h}^{-1}$, respectivamente, podendo estar relacionado aos baixos valores de umidade para os solos avaliados. Os menores valores, que estiveram bem próximos à origem do gráfico, tiveram sua relação vinculada aos Latossolos Vermelho distrófico típico com pastagem natural.

O modelo teórico de Green-Ampt teve seus parâmetros ajustados, conforme Tabela 4, com boa representatividade, para a infiltração potencial.

Tabela 4. Relação entre tipo de solo, cobertura vegetal, declive, intensidade de precipitação (IP) e os parâmetros condutividade hidráulica do solo saturado (Ks), potencial matricial médio na frente de umedecimento ( $\Psi$ f), umidade inicial do solo $(\theta \mathrm{i})$ e umidade do solo na saturação $(\theta \mathrm{s})$ da equação de Green-Ampt com o índice de representatividade do ajuste $\left(\mathrm{R}^{2}\right)$.

\begin{tabular}{|c|c|c|c|c|c|c|}
\hline Parcela & $\operatorname{IP}\left(\mathrm{mm} \mathrm{h}^{-1}\right)$ & $\mathrm{Ks}\left(\mathrm{mm} \mathrm{h}^{-1}\right)$ & $\Psi f(\mathrm{~cm})$ & $\theta \mathrm{i}\left(\mathrm{cm}^{\left.-\mathrm{cm}^{-3}\right)}\right.$ & $\theta \mathrm{s}\left(\mathrm{cm} \cdot \mathrm{cm}^{-3}\right)$ & $\mathbf{R}^{2}$ \\
\hline \multirow{5}{*}{$\begin{array}{c}\mathrm{LVd}, \mathrm{PN} \text { e } \\
10 \% \text { de declive } \\
\quad(\mathrm{P} 1)\end{array}$} & 30 & 0,9846 & 1,9878 & 0,3310 & 0,4642 & 1,0000 \\
\hline & 60 & 0,9963 & 1,9985 & 0,3965 & 0,5281 & 1,0000 \\
\hline & 90 & 0,9955 & 1,9569 & 0,1689 & 0,4249 & 1,0000 \\
\hline & 120 & 0,9971 & 1,9648 & 0,1689 & 0,4416 & 1,0000 \\
\hline & 150 & 0,9946 & 1,9936 & 0,2984 & 0,4478 & 1,0000 \\
\hline \multirow{5}{*}{$\begin{array}{c}\text { LVd, PN e } \\
13 \% \text { de declive } \\
\text { (P2) }\end{array}$} & 30 & 0,9965 & 1,9832 & 0,3815 & 0,4046 & 0,9344 \\
\hline & 60 & 0,9965 & 1,9756 & 0,4708 & 0,5285 & 0,9997 \\
\hline & 90 & 0,9965 & 1,9563 & 0,3725 & 0,6630 & 0,9233 \\
\hline & 120 & 0,9965 & 1,9654 & 0,3376 & 0,4822 & 1,0000 \\
\hline & 150 & 0,9965 & 1,9430 & 0,4142 & 0,6361 & 1,0000 \\
\hline \multirow{5}{*}{$\begin{array}{c}\text { Cd, PND e } \\
15 \% \text { de declive } \\
\text { (P3) }\end{array}$} & 30 & 0,9923 & 1,9423 & 0,3995 & 0,4575 & 1,0000 \\
\hline & 60 & 0,9962 & 1,9236 & 0,3767 & 0,4594 & 1,0000 \\
\hline & 90 & 0,9899 & 1,9632 & 0,4505 & 0,4770 & 1,0000 \\
\hline & 120 & 0,9965 & 1,9681 & 0,3290 & 0,5751 & 1,0000 \\
\hline & 150 & 0,9863 & 1,9529 & 0,2601 & 0,5847 & 1,0000 \\
\hline \multirow{5}{*}{$\begin{array}{l}\text { LVAd, PN e } \\
13 \% \text { de declive } \\
\text { (P4) }\end{array}$} & 30 & 0,9911 & 2,0000 & 0,4390 & 0,4926 & 1,0000 \\
\hline & 60 & 0,9950 & 1,9560 & 0,3748 & 0,5260 & 1,0000 \\
\hline & 90 & 0,9909 & 1,5263 & 0,3594 & 0,5103 & 1,0000 \\
\hline & 120 & 0,9963 & 1,8700 & 0,3281 & 0,4789 & 1,0000 \\
\hline & 150 & 0,9881 & 1,9321 & 0,2333 & 0,4622 & 1,0000 \\
\hline \multirow{5}{*}{$\begin{array}{l}\text { LVAd, MN e } \\
15 \% \text { de declive } \\
\text { (P5) }\end{array}$} & 30 & 0,4666 & 1,9999 & 0,5351 & 0,5872 & 1,0000 \\
\hline & 60 & 0,9963 & 1,9753 & 0,4700 & 0,5114 & 1,0000 \\
\hline & 90 & 0,9955 & 1,9628 & 0,4266 & 0,5114 & 1,0000 \\
\hline & 120 & 0,9971 & 1,9854 & 0,4218 & 0,5114 & 1,0000 \\
\hline & 150 & 0,9946 & 1,9912 & 0,3807 & 0,5114 & 1,0000 \\
\hline
\end{tabular}

Fonte: Elaboração dos autores. 
Procurou-se também avaliar neste estudo o de armazenamento $(\Delta \mathrm{A})$ no perfil monitorado $(0$ desempenho do solo e da infiltração relacionando a a $400 \mathrm{~mm}$ ). Na Tabela 5 pode-se observar que o infiltração observada (Iobs) em campo e a variação processo de infiltração predominou para a maioria dos testes realizados.

Tabela 5. Relações entre intensidade de precipitação (IP), tipo de solos, cobertura vegetal, declive (d), umidade inicial $(\theta i)$ e a performance da infiltração observada (Iobs) em relação à variação de armazenamento no perfil de solo avaliado $(\Delta \mathrm{A})$.

\begin{tabular}{|c|c|c|c|c|}
\hline IP $\left(\mathrm{mm} \mathrm{h}^{-1}\right)$ & Parcela & $\theta \mathrm{i}\left(\mathrm{cm}^{3} \cdot \mathrm{cm}^{-3}\right)$ & Iobs/ $\Delta \mathrm{A}$ & Performance \\
\hline \multirow{5}{*}{30} & LVd, PN, $10 \% \mathrm{~d}$ & 0,3532 & 1,0166 & Infiltração \\
\hline & $\mathrm{LVd}, \mathrm{PN}, 13 \% \mathrm{~d}$ & 0,4232 & 1,0476 & Infiltração \\
\hline & $\mathrm{Cd}, \mathrm{PND}, 15 \% \mathrm{~d}$ & 0,1802 & 1,5894 & Infiltração \\
\hline & LVAd, PN, $10 \% \mathrm{~d}$ & 0,1802 & 1,7193 & Infiltração \\
\hline & LVAd, MN, $15 \% \mathrm{~d}$ & 0,3185 & 2,4889 & Infiltração \\
\hline \multirow{5}{*}{60} & LVd, PN, $10 \% \mathrm{~d}$ & 0,3729 & 0,8619 & Armazenamento \\
\hline & $\mathrm{LVd}, \mathrm{PN}, 13 \% \mathrm{~d}$ & 0,4602 & 2,4532 & Infiltração \\
\hline & $\mathrm{Cd}, \mathrm{PND}, 15 \% \mathrm{~d}$ & 0,3641 & 1,1575 & Infiltração \\
\hline & LVAd, PN, $10 \% \mathrm{~d}$ & 0,3300 & 1,1266 & Infiltração \\
\hline & LVAd, MN, $15 \% \mathrm{~d}$ & 0,4048 & 2,8552 & Infiltração \\
\hline \multirow{5}{*}{90} & LVd, PN, $10 \% \mathrm{~d}$ & 0,3995 & 0,8228 & Armazenamento \\
\hline & LVd, PN, $13 \% \mathrm{~d}$ & 0,3767 & 0,4977 & Armazenamento \\
\hline & $\mathrm{Cd}, \mathrm{PND}, 15 \% \mathrm{~d}$ & 0,4505 & 3,8172 & Infiltração \\
\hline & LVAd, PN, $10 \% \mathrm{~d}$ & 0,3290 & 2,4745 & Infiltração \\
\hline & LVAd, MN, $15 \% \mathrm{~d}$ & 0,2601 & 1,1086 & Infiltração \\
\hline \multirow{5}{*}{120} & LVd, PN, $10 \% \mathrm{~d}$ & 0,3835 & 1,1450 & Infiltração \\
\hline & $\mathrm{LVd}, \mathrm{PN}, 13 \% \mathrm{~d}$ & 0,3748 & 1,7898 & Infiltração \\
\hline & $\mathrm{Cd}, \mathrm{PND}, 15 \% \mathrm{~d}$ & 0,3677 & 1,5532 & Infiltração \\
\hline & LVAd, PN, $10 \% \mathrm{~d}$ & 0,3356 & 1,9974 & Infiltração \\
\hline & LVAd, MN, $15 \% \mathrm{~d}$ & 0,2387 & 4,8285 & Infiltração \\
\hline \multirow{5}{*}{150} & LVd, PN, $10 \% \mathrm{~d}$ & 0,5014 & 3,8910 & Infiltração \\
\hline & $\mathrm{LVd}, \mathrm{PN}, 13 \% \mathrm{~d}$ & 0,4404 & 2,0605 & Infiltração \\
\hline & $\mathrm{Cd}, \mathrm{PND}, 15 \% \mathrm{~d}$ & 0,3997 & 1,8761 & Infiltração \\
\hline & LVAd, PN, $10 \% \mathrm{~d}$ & 0,3953 & 2,0278 & Infiltração \\
\hline & LVAd, MN, $15 \% \mathrm{~d}$ & 0,3567 & 3,1940 & Infiltração \\
\hline
\end{tabular}

Fonte: Elaboração dos autores.

Verificou-se também, que para os Latossolos Vermelho distrófico, para intensidades de 60 e $90 \mathrm{~mm} \mathrm{~h}^{-1}$, obtiveram desempenho favorável ao armazenamento sobre os demais. Este fato pode se relacionar com os baixos valores de umidades iniciais associadas a sua textura, volume total de poros.

\section{Conclusão}

Pode-se concluir que dentro dos limites da aplicabilidade e das respectivas limitações de cada modelo, todos forneceram bons coeficientes de determinação para os Latossolos Vermelho distrófico típico, Cambissolo distrófico e Latossolos Vermelho Amarelo distrófico, cobertura vegetal de pastagem nativa, pastagem nativa degradada e mata nativa, respectivamente.

Dentro de uma mesma classe de solo, a cobertura vegetal, a declividade, os atributos físicos e hídricos do solo, a intensidade de precipitação e a umidade 
inicial influenciaram nos parâmetros das equações.

O modelo de Kostiakov, Kostiakov-Lewis e Horton, ficaram próximos do linear para todos os eventos de precipitação, tipos de solos e cobertura vegetal.

Os Latossolos Vermelho distrófico, para intensidades de precipitação de 60 e $90 \mathrm{~mm}$ $\mathrm{h}^{-1}$, obtiveram a performance favorável ao armazenamento de água no solo sobre os demais.

Todos os solos mostraram semelhança no comportamento da infiltração inicial exceto $o$ Latossolo Vermelho distrófico típico com pastagem nativa e $13 \%$ de umidade.

Os valores de tempo de saturação ficaram próximos à origem do gráfico para quase todos os eventos de precipitação.

\section{Referências}

BAGATINI, T.; COGO, N. P.; GILlES, L.; PORTELA, J. C.; PORTZ, G.; QUEIROZ, H. T. Perdas de solo e água por erosão hídrica após mudança no tipo de uso da terra, em dois métodos de preparo do solo e dois tipos de adubação. Revista Brasileira de Ciência do Solo, Viçosa, v. 35, n. 3, p. 999-1011, maio/jun. 2011.

BERNARDO, S.; SOARES, A. A.; MANTOVANI, E. C. Manual de irrigação. 8. ed. Viçosa: UFV, 2006. 625 p.

BEUTLER, A. N.; SILVA, M. L. N.; CURI, N.; FERREIRA, M. M.; CRUZ; J. C.; PEREIRA FILHO, I. A. Resistência à penetração e permeabilidade de latossolo vermelho distrófico típico sob sistemas de manejo na região dos cerrados. Revista Brasileira de Ciência do Solo, v. 25, n. 1, p. 166-177, 2001.

BEZERRA, S. A.; CANTALICE, J. R. B. Influência da cobertura do solo nas perdas de água e desagregação do solo em entressulcos. Revista Caatinga, Mossoró, v. 22, n. 3, p. 18-28, jul./set. 2009.

CECÍlIO, R. A.; SILVA, D. D.; PRUSKI, F. F.; MARTINEZ, M. A. Modelagem da infiltração de água no solo sob condições de estratificação utilizandose a equação de Green-Ampt. Revista Brasileira de Engenharia Agrícola e Ambiental, Campina Grande, v. 7, n. 3, p. 415-422, set./dez. 2003.

CUNHA, J. L. X. L.; ALBUQUERQUE, A. W.; SILVA, C. A.; ARAÚJO, E.; SANTOS, R. B. J. Velocidade de
Infiltração da água em um latossolo amarelo submetido ao sistema de manejo plantio direto. Revista Caatinga, Mossoró, v. 22, n. 1, p. 199-205, jan./mar. 2009.

DALRI, A. B.; CORTEZ, G. E. P.; RIUL, L. G. S.; ARAÚJO, J. A. C.; CRUZ, R. L. Influência da aplicação de vinhaça na capacidade de infiltração de um solo de textura franco arenosa. Irriga, Botucatu, v. 15, n. 4, p. 344-352, out./dez. 2010.

FERREIRA, A. O.; GONZATTO, R.; MIOLA, A.; ELTZ, F. L. F.; AMADO, T. J. C. Influência da declividade e de níveis de cobertura do solo no processo de erosão com chuva simulada. Revista Verde, Mossoró, v. 5, n. 5, p. 182-190, dez. 2010. Edição Especial.

GIAROLA, N. F. B.; CURI, N.; SIQUEIRA, J. O.; CHAGAS, C. S.; FERREIRA, M. M. Solos na região sob Influência do reservatório da hidrelétrica de itutinga, Camargos $(M G)$ : perspectiva ambiental. Belo Horizonte: UFLA/CEMIG, 1997. 101 p.

GOMES, N. M. Variabilidade espacial de atributos fisico-hídricosdo solo da sub-bacia hidrográfica do RibeirãoMarcela na Região do Alto Rio Grande, $M G$. 2005. Dissertação (Mestrado em Irrigação e Drenagem) Universidade Federal de Lavras, Lavras.

GREEN, W. H.; AMPT, G. A. Studies on soil physics-1. The flow of air and water through soils. Journal of Agricultural Science, Cambridge, v. 4, n. 1, p. 1-24, 1911.

HORTON, R. E. An approach toward a physical interpretation - capacity. Soil Science Society of America Proceeding, Madison, v. 5, p. 399-417, 1940.

KÖPPEN, W. Das geographische system der klimatologie. Berlim: Borntrager. 1936. 44 p.

KOSTIAKOV, A. N. On the dynamics of the coefficient of water percolation in soils and the necessity of studying it from dynamic point of view for purposes of amelioration. In: 6th Committee of International Society of Soil Science. Paris: International Soil Science Society, 1932. v. 6, p. 17-21.

PAIXÃO, F. J. R.; ANDRADE, A. R. S.; AZEVEDO, C. A. V.; COSTA, T. L.; GUERRA, H. O. C. Ajuste da curva de infiltração por meio de diferentes modelos empíricos. Pesquisa Aplicada \&Agrotecnologia, Guarapuava, v. 2, n. 1, p. 108-112, jan./abr. 2009.

PANACHUKI, E.; BERTOL, I.; SOBRINHO, T. A.; OLIVEIRA, P. T. S.; RODRIGUES, D. B. B. Perdas de solo e de água e infiltração de água em latossolo vermelho sob sistemas de manejo. Revista Brasileira de Ciência do Solo, Viçosa, v. 35, n. 5, p. 1777-1786, set./out. 2011.

PINHEIRO, A.; TEIXEIRA, L. P.; KAUFMANN, V. Capacidade de infiltração de água em solos sob diferentes 
usos e práticas de manejo agrícola. Revista Ambiente e Água, Taubaté, v. 4, n. 2, p. 188-199, 2009.

SILVA, A. M.; OLIVEIRA, P. M.; MELLO, C. R.; PIERANGELI, C. Vazões mínimas e de referência para outorga na região do Alto Rio Grande, MG. Revista Brasileira de Engenharia Agrícola e Ambiental, Campina Grande, v. 10, n. 2, p. 374-380, abr.jun. 2006.

SILVA, J. V.; ALECRIM, M. A. B.; SILVA, D. O.; COSTA, C. C.; OLIVEIRA, R. J. Perdas de solo e água por erosão hídrica em floresta equiânea em um Latossolo Vermelho-Amarelo. Revista Brasileira de Ciências Agrárias, Recife, v. 5, n. 4, p. 579-584, out./dez. 2010.
SILVA, P. M. O. Modelagem do escoamento superficial e da erosão na sub-bacia hidrográfica do ribeirão Marcela, Alto Rio Grande, MG. 2006. Tese (Doutorado em Engenharia Agrícola) - Universidade Federal de Lavras, Lavras.

URCHEI, M. A.; FIETZ, C. R. Infiltração de água em um latossolo roxo muito argiloso em dois sistemas de manejo. Irriga, Botucatu, v. 7, n. 1, p. 1-9, 2002.

WEILER, M.; NAEF, F. An experimental tracer study on the role of macropores in infiltration in grassland soils. Hydrological Processes, Malden, v. 17, n. 2, p. 477-494, fev. 2003. 